\title{
TRANSCRIPTOME PROFILING OF HALOXYLON AMMODENDRON SEEDLING AT LOW TEMPERATURE CONDITION
}

\author{
Peng, M. W. - ChAng, Y. L. - WANG, M. ${ }^{*}$ - ChU, G. M. ${ }^{*}$ \\ Agricultural College, Shihezi University \\ Road of North 4th, Shihezi City, Xinjiang 832003, China \\ *Corresponding authors \\ e-mail:wangm1205@163.com,chgmxj@163.com \\ (Received $20^{\text {th }}$ Oct 2018; accepted $7^{\text {th }}$ Jan 2019)
}

\begin{abstract}
Haloxylon ammodendron, a chenopodiaceae shrub species that mainly distributes in northwest of China, is an ecologically important foundation species and exhibits substantial low-temperature tolerance in the desert area. We employed RNA-Seq technologies to identify genes involved in low temperature, and identified 17995, 15511 and 1550 differentially expressed genes from the comparison of chilling treatment versus control, freezing treatment versus control and freezing treatment versus chilling treatment, respectively. By performing BLAST analysis of these unigenes against public databases, "single-organism metabolic process" and "membrane" were strongly affected at low temperature, and "Plant hormone signal transduction" pathway played an important role in resistance of the plants. In addition, we analyzed transcription factors, and found 499, 453, 55 of them were differentially expressed in these three comparison groups, respectively. A large number of transcription factors were identified at low temperature, and six transcription factors were selected for further expression analysis using qRTPCR. This study can understand the molecular mechanisms of Haloxylon ammodendron at low temperature condition, and also can serve as a valuable resource for relevant research on low-temperature tolerance.
\end{abstract}

Keywords: Haloxylon ammodendron, chilling tolerance, freezing tolerance, transcription factor, illumina

\section{Introduction}

Temperature is one of the important environmental factors, and the normal physiological and metabolic activities of plants need suitable temperature. Low temperature restricts the distribution of plants by influencing their germination, growth and development, it can cause adverse effects and even can lead to death (Cai et al., 2011). However, some species have high tolerance to low temperature condition, especially some desert plants that grow in harsh environment. Haloxylon ammodendron is mainly distributes in the desert and semi desert areas of the Junggar basin in Xinjiang, China. As the dominant species in the desert area, it has not only ecological value but also economic value. However, the habitat of the Junggar basin is abominable that the maximum temperature is above $40{ }^{\circ} \mathrm{C}$, and the minimum temperature is around $-40{ }^{\circ} \mathrm{C}$. It is especially interesting that some desert plants have low temperature germination feature during snow melting in early spring (Huang, 2002; Wang et al., 2006; Huang et al., 2003; Zhang, 2010; Han et al., 2011; Zhou, 2016; Peng, 2018), these desert plants include H. ammodendron, Haloxylon Persicum, Anabasis elatior, Lepidium apetalum and Anabasis aphylla. Huang research showed that when the early spring temperature rises and the snow melts, which will provide water supply for seed germination, and also provide a favorable environmental foundation for plant breeding (Huang, 2002). Similarly, Suaeda physophora can germinate at $10{ }^{\circ} \mathrm{C}$ and even at $0{ }^{\circ} \mathrm{C}$ from field 
observation ( $\mathrm{Li}$ and Zhang, 2007). In addition, Wang studied proteomics and metabolomics of $H$. ammodendron and Anabasis aphylla seedlings under low temperature (Wang, 2018). Therefore, we analyzed the transcription mechanism of $H$. ammodendron at low temperature condition, it will provide valuable resource for relevant research on low-temperature tolerance.

Transcriptional studies, with all the transcriptional information as the research object, analyze the regulatory network of plant bodies from the whole, it can also carry out quantitative and network analysis of individual genes or several genes (Cheng et al., 2015). A scholar had conducted the transcriptome sequencing of Ammopiptanthus mongolicus, and obtained a series of annotated genes and differentially expressed genes at low temperature condition, moreover, some metabolic pathways and transcription factors were also studied in order to understand the mechanism of frost resistance (Pang et al., 2013). Some studies presented the whole transcriptome of Camellia sinensis and Sophora moorcroftiana, and analyzed the change of genes expression during cold acclimation, these information provide a basis for plants to increase tolerance and the ability to adapt to the environment (Wang et al., 2013; Li et al., 2015). Chen et al. identified a large number of cold-responsive genes of Populus euphratica, and indicated that the transcription factor, $\mathrm{ABA}$ and calcium signal transduction may play an important role at low temperature condition (Chen et al., 2014). Our study presents the transcriptome profile of $H$. ammodendron seedling using Illumina sequencing technology, and yields insights into the molecular mechanisms of $H$. ammodendron seedling at low temperature condition. It could help to explore some genes related to low temperature and improve the understanding of low-temperature tolerance and plantenvironment interactions.

\section{Materials and methods}

\section{Location of $\mathrm{H}$. ammodendron}

H. ammodendron was located on the desert-oasis ecotone spanning an elevation range of 258-265 $\mathrm{m}$ in South Junggar Basin, NW China $\left(45^{\circ} 22^{\prime} 43.4^{\prime \prime} \mathrm{N}, 84^{\circ} 50^{\prime} 32.5^{\prime \prime} \mathrm{E}\right)$, this is a transitional zone from the oasis to desert (Fig. 1). Geological substrates include aeolian deposits sandy soil and highly eroded diluvial soil. The annual mean temperature varies from 5 to $9{ }^{\circ} \mathrm{C}$, and minimum winter temperature varies from -30 to $41^{\circ} \mathrm{C}$ and maximum summer temperatures are $30-40{ }^{\circ} \mathrm{C}$. Snow-melting appears at the end of winter, together with the rainfall that occurs in summer and annual precipitation amounts from $100 \mathrm{~mm}$ to $250 \mathrm{~mm}$. Annual potential evaporation is greater than 2000 mm (Wang et al., 2014).

\section{Experimental materials and low temperature treatment}

The research material is the seedling of $H$. ammodendron. Experimental seeds were collected in October 2016 from Xinjiang, the southern margin of the Junggar Basin. These seeds were cultured in Petri dish with distilled water. When the length of the seedling is $3 \mathrm{~cm}$, we treated the seedlings with different temperature. Based on the germination characteristics of $H$. ammodendron seeds under the freezing and thawing conditions in early spring (Wang et al., 2006; Huang et al., 2003), and the strong adaptability of the seedlings to environment temperature, we set three temperature treatments, control $\left(20^{\circ} \mathrm{C}\right)$, chilling treatment $\left(3^{\circ} \mathrm{C}\right)$ and freezing treatment $\left(-3^{\circ} \mathrm{C}\right)$, 
respectively. Finally, all samples were preserved using liquid $\mathrm{N}$ and sent to the Beijing Compass Biotechnology Co., Ltd. to perform high throughput sequencing.

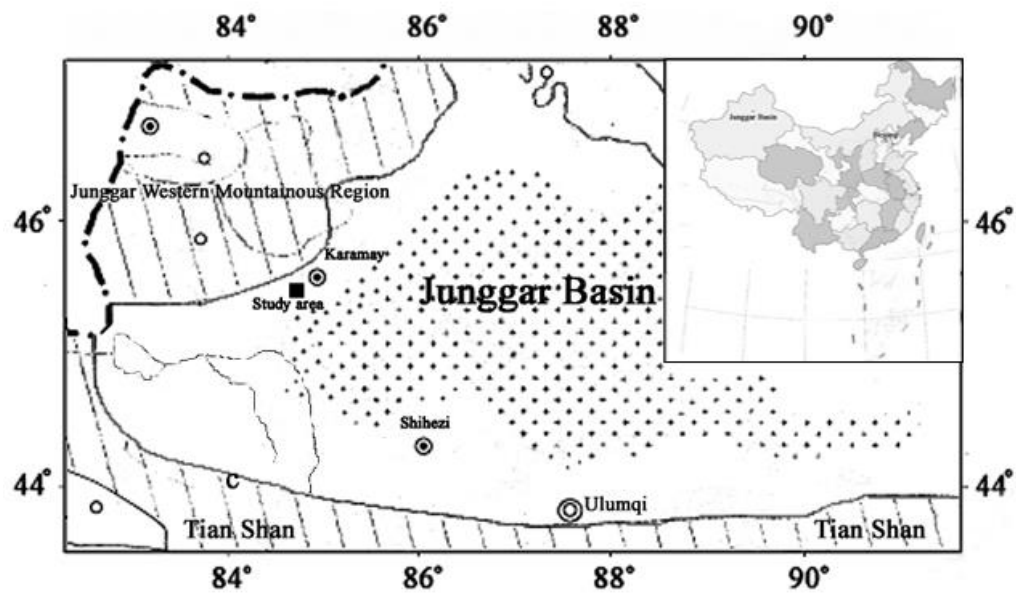

Figure 1. The location map of the Junggar Basin

\section{RNA extraction and cDNA library construction}

RNA extracted from control samples and treatment samples was further tested by Agilent 2100 Bioanalyzer (Agilent Technologies, CA, USA). After testing the RNA samples, the eukaryotic mRNA was enriched with magnetic beads containing Oligo (dT). Then join fragmentation buffer to break mRNA into short segments. Using mRNA as template, a base random primer was used to synthesize a chain of cDNA. Then buffer solution was added to synthesise second cDNA chains, after purification, elution, terminal repair, junction sequencing and PCR amplification, the library was finally completed. The sequencing library was sequenced by Illumina Hiseq 2500.

\section{Sequencing, de novo assembly and functional annotation}

High quality RNA samples were constructed from the sequencing library. Transcriptome sequencing of the library was carried out using the Illumina Hiseq 2500 high throughput sequencing platform (HiSeq/Miseq), and base calling is used to transform the original image data after sequencing into sequence data, that is the raw reads. Raw reads uses sliding window method to remove redundant sequence, sequence of poor quality, and sequence containing N. After screening, the high quality short sequence (clean reads) is obtained, and the follow-up analysis is carried out by this short sequence.

The high quality short sequence after removing the sequence of the joint, and the low quality at both ends and the low complex sequence was de novo assembled by using Trinity (Grabherr et al., 2011; Haas et al., 2009). BLAST software (Avagyan, 2009) is used to transform the transcript sequence into a protein sequence and then function annotation with multiple databases, and functional annotations include $\mathrm{Nr}$, Pfam (Bateman et al., 2000), Swiss-Prot (Consortium, 2015), KOG (Tatusov et al., 2003), Nt, GO (Harris et al., 2004) and KEGG databases. In addition, the software used in NR, NT, Swiss-Prot and KOG database is NCBI blast 2.3.0+. Pfam database is HMMER 
package, GO function annotation software is Blast2GO v2.5 (Conesa et al., 2005), and KEGG annotation software is KOBAS 2.1.1 (Xie et al., 2011).

\section{Quantitative real-time PCR analysis}

In order to validate the reliability of RNA-Seq and DGE experiment, six transcripts were selected for quantitative qRT-PCR test. Total RNAs were quantified by the NanoDrop ND-2000 (Thermo Scientific) and the RNAs integrity was assessed using agarose gel electrophoresis. While QC of RNA was qualified, in turn to treat RNA of DNaseI, then reverse transcription, cDNA dilution, last PCR by Applied Biosystems 7900HT PCR. After completion of the reaction the data analysis based on the obtained sample $\mathrm{Ct}$ value. Relative gene expression levels were calculated using the $2^{-\Delta \Delta \mathrm{Ct}}$ (Livaka and Schmittgen, 2001).

\section{Results}

\section{Transcriptome sequencing and assembly}

Transcriptome sequences are valuable resources. A cDNA library from a seedling of $H$. ammodendron was constructed and sequenced using the Illumina HiSeq2500 platform. Total clean bases generated from each sample exceeded 12G. Total of 40535462 raw reads were generated for the control sample (CK). After removing lowquality reads and trimming off the adapter sequences, 40211335 clean reads were obtained. The Q20 bases percentage and the Q30 bases percentage were $95.40 \%$ and $89.26 \%$, respectively. Similar to the control, we obtained 41432865 raw reads and 40994453 clean reads from chilling sample at $3{ }^{\circ} \mathrm{C}(\mathrm{CA})$, and obtained 43214542 raw reads and 42973332 clean reads from freezing sample at $-3{ }^{\circ} \mathrm{C}(\mathrm{CB})$. The Q20 percentage of two samples (CA and CB) were exceeded 94\% and Q30 were exceeded $88 \%$ (Table 1).

Using Trinity, all the total reads were mixed for further de novo assembly, and these produced 437604 transcripts with an N50 of $1268 \mathrm{bp}$ and an average length of 764.78 bp. In addition, all the high-quality reads were assembled into 257695 unigenes with an N50 of $713 \mathrm{bp}$ and an average length of $546.93 \mathrm{bp}$ (Table 2).

Table 1. Sequencing statistics of the H. ammodendron

\begin{tabular}{c|c|c|c|c|c|c|c}
\hline Sample & $\begin{array}{c}\text { Raw bases } \\
(\mathbf{G})\end{array}$ & $\begin{array}{c}\text { Clean bases } \\
(\mathbf{G})\end{array}$ & Raw reads & Clean reads & $\mathbf{Q 2 0}(\boldsymbol{\%})$ & $\mathbf{Q 3 0}(\boldsymbol{\%})$ & $\begin{array}{c}\text { GC content } \\
(\boldsymbol{\%})\end{array}$ \\
\hline CK & 12.16 & 12.06 & 40535462 & 40211335 & 95.40 & 89.26 & 41.68 \\
CA & 12.43 & 12.30 & 41432865 & 40994453 & 95.26 & 89.02 & 41.10 \\
CB & 12.97 & 12.89 & 43214542 & 42973332 & 94.99 & 88.54 & 41.33 \\
\hline
\end{tabular}

Table 2. Summary statistics of assembly of $H$. ammodendron transcriptome

\begin{tabular}{c|c|c|c|c}
\hline & Total number & Total assembled bases & N50 (bp) & Average length (bp) \\
\hline Transcripts & 437604 & 334670343 & 1268 & 764.78 \\
Unigenes & 257695 & 140940214 & 713 & 546.93 \\
\hline
\end{tabular}




\section{Blast analysis}

To predict and analyze the function of the assembled transcripts, non-redundant sequences were submitted to a BLASTx search against the following databases: $\mathrm{Nr}$ (NCBI non-redundant protein sequences), Pfam (Protein family), Swiss-Prot (a manually annotated and reviewed protein sequence database), KOG (eukaryotic orthologous groups), Nt (NCBI non-redundant nucleotide sequences), GO (Gene Ontology) and KEGG (Kyoto Encyclopedia of Genes and Genomes). The blast results show that the number of unigenes annotated in the Nr, Pfam, Swiss-Prot, KOG, Nt, GO, KEGG database was 495669 (41.41\%), 23 (0.02\%), 30748 (25.69\%), 29867 (24.95\%), 27693 (23.13\%), 21179 (17.69\%), 45557 (38.06\%), respectively (Table 3).

Table 3. Summary statistics of annotation of all unigenes

\begin{tabular}{c|c|c|c|c|c|c|c|c}
\hline Database & Nr & PFAM & Swiss-prot & KOG & Nt & GO & KEGG & ALL \\
\hline Annotated number & 49566 & 23 & 30748 & 29867 & 27693 & 21179 & 45557 & 119682 \\
Annotated percentage (\%) & 41.41 & 0.02 & 25.69 & 24.95 & 23.13 & 17.69 & 38.06 & 100 \\
\hline
\end{tabular}

By the Venn diagram, the number of unigenes annotated in $\mathrm{Nr}$ and $\mathrm{GO}$ databases is large, 10455 and 21179, respectively. The number of unigenes annotated only in KOG and Swiss-prot databases is very few, 709 and 25, respectively. There are 2450 unigenes annotated to the Nt database. A total of 119682 unigenes are annotated, and the number of unigenes annotated to 5 databases was 0 (Fig. 2).

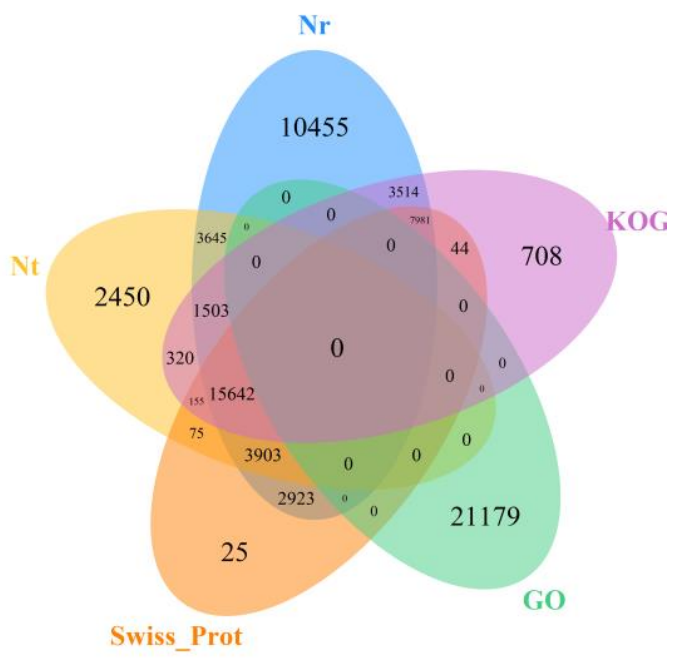

Figure 2. Venn diagram of unigenes were annotated in five databases

\section{Functional annotation of $\mathrm{H}$. ammodendron transcriptome sequences}

A BLASTx search of protein sequences from the National Center for Biotechnology Information $\mathrm{nr}$ database using the unigenes and E-value distribution was performed (Fig. $3 A$ and $B$ ), and the resulting E-value distribution revealed that $49.65 \%$ of the unigene sequences had top matches. The result shows $80.44 \%$ of the unigene sequences matching with specific protein was more than $60 \%$ in similarity distribution. To study 
the sequence conservation of $H$. ammodendron in other plant species, we analyzed the species distribution of the All-Unigene datasets. The results show that $51.26 \%$ of the distinct sequences have top matches with the sequences from Beta vulgaris subsp. Vulgaris (51.25\%), followed by Spinacia oleracea (31.29\%), Vitis vinifera (1.39\%), Daucus carota subsp. sativus (0.65\%) and other (15.42\%) (Fig. 3C).

A

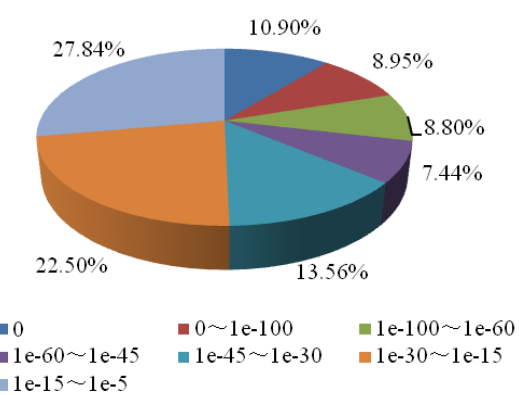

$\mathbf{C}$
B

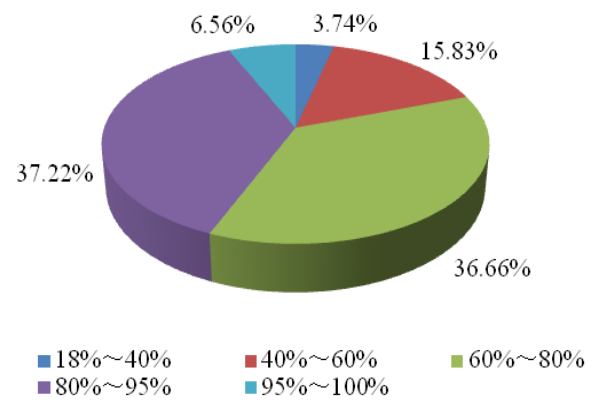

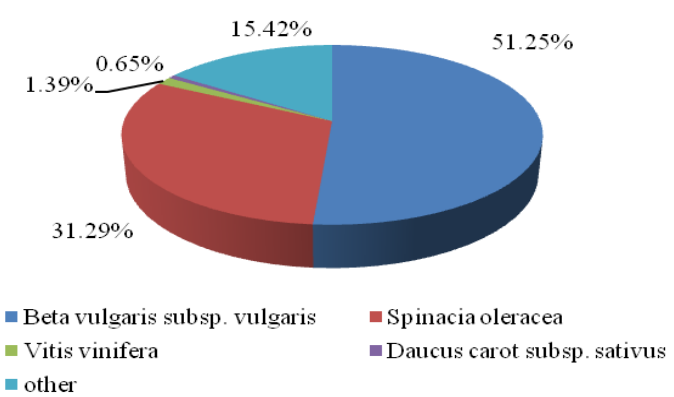

Figure 3. Species distribution of BLASTx results. Unigenes were used in a BLASTx search of the $n r$ database $(\boldsymbol{A})$ E-value distribution; $(\boldsymbol{B})$ similarity distribution; $(\boldsymbol{C})$ species distribution

Gene Ontology (GO) assignments were used to classify the functions of the predicted H. ammodendron genes, 21179 (17.69\%) unigenes were classified into three major function categories (Biological Process, Cellular Component and Molecular Function). In terms of Biological Processes 14019 unigenes were annotated, the top three GO terms were "single-organism metabolic process" (1531), "cellular protein modification process" (982) and "phosphate-containing compound metabolic process" (880). In terms of cellular components, there are 12639 unigenes were annotated, the top three GO terms were "intrinsic component of membrane" (6109), "intracellular membranebounded organelle" (2060) and "membrane" (1332). In terms of molecular function, there are 16176 unigenes were annotated, the top three GO terms were "purine ribonucleoside binding" (3100), "transition metal ion binding" (1507) and "nucleic acid binding" (1399) (Fig. 4).

To assess the integrality of our transcriptome library and effectiveness of the annotation process, we aligned the all-unigenes to the KOG database and 29867 were identified. By classifying the possible functions of these unigenes, they were grouped into 25 functional categories. The clusters related to "general function prediction only" (6584) were the largest group, followed by "signal transduction mechanisms" (3948), "posttranslational modification, protein turnover, chaperones" (3541), and “carbohydrate transport and metabolism" (2052) (Fig. 5). 


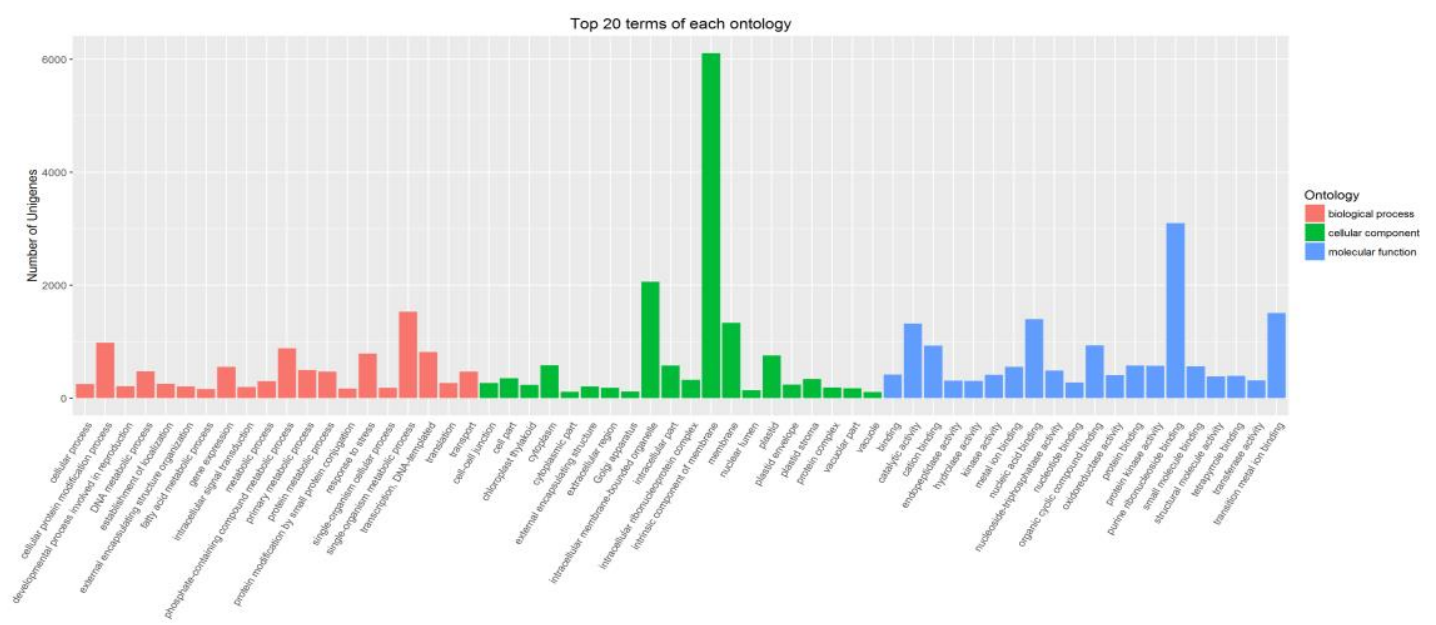

Figure 4. Gene ontology (GO) functional classification of the unigenes

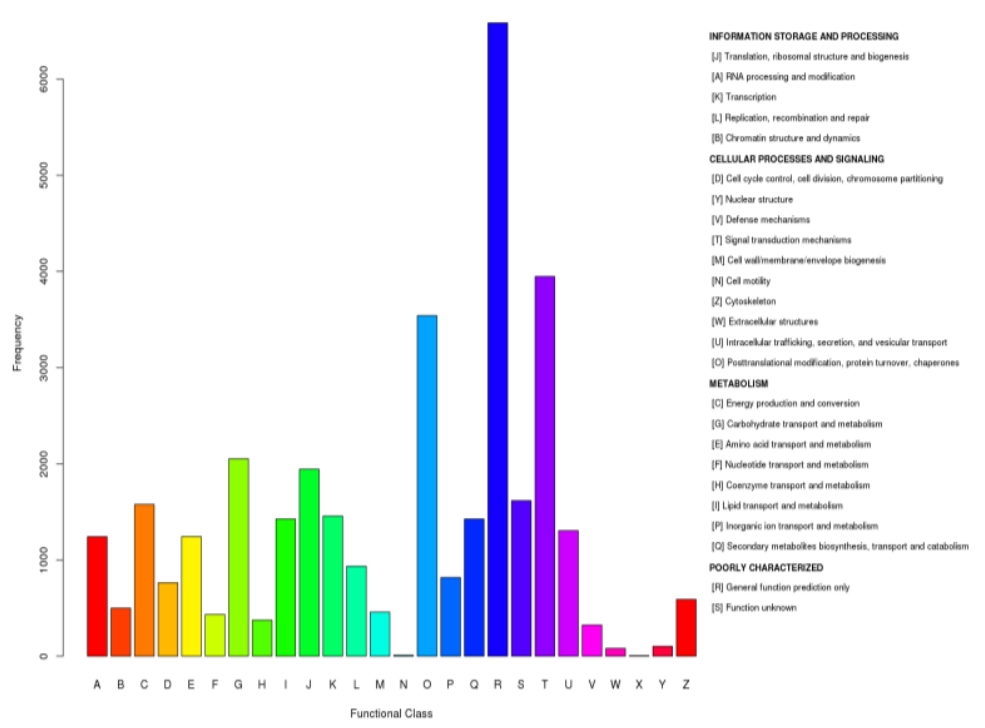

Figure 5. Eukaryotic ortholog groups (KOG) functional classification of the unigenes

To identify the biological pathways in the annotated sequences using the Kyoto Encyclopedia of Genes and Genomes (KEGG), the assembled unigenes were assigned to five specific pathways, including Cellular Processes (A), Environmental Information Processing (B), Genetic Information Processing (C), Metabolism (D), and Organism Systems (E). Metabolism was the category with the greatest number of genes (9561), with carbohydrate metabolism and overview as the two pathways represented by the most genes. 1718 were related to translation, 1507 were related to signal transduction, 1357 were related to folding, sorting and degradation (Fig. 6).

\section{Genes differentially expressed among the samples}

To examine the transcript alterations occurring in the germination exposed to low temperature, we used the RPKM method to calculate the expression levels of the unigenes and to identify differentially expressed genes between the types of samples. 
The expression of a high number of unigenes was affected at low temperature condition. Only the genes whose expression was identified as being significantly changed with the FDR $<0.05$ were retained. To analyze the similarities and differences among the lowtemperature responsive transcriptome, a hierarchical clustering was prepared to replicates of the control $(\mathrm{CK})$, chilling treatment $(\mathrm{CA})$ and freezing treatment $(\mathrm{CB})$. When the differentially expressed genes were compared under the three conditions, we discovered 17995, 15511 and 1550 genes in the comparisons of CA versus CK, CB versus $\mathrm{CK}$ and $\mathrm{CB}$ versus $\mathrm{CA}$, respectively. Among these, the genes with significant difference $(|\log F C|>1)$ are displayed in Figure 7.

\section{Functional classification of the differentially expressed genes by gene ontology analysis}

To identify the genes that are differentially expressed at low temperature treatment, a functional categorization was carried out by GO analysis. By comparing CA and CK, 17995 differentially expressed genes, including 9286 down-regulated genes and 8709 up-regulated genes, revealed by differentially expressed genes analysis were functionally assigned to the relevant terms in three categories (Biological Process, Cellular Component, and Molecular Function) of the GO database. Among these groups, the terms "single-organism metabolic process", "membrane", and "metal ion binding" were dominant in each of the three main categories, respectively (Fig. Al). By comparing $\mathrm{CB}$ and $\mathrm{CK}, 15511$ differentially expressed genes, including 7915 downregulated genes and 7596 up-regulated genes, were functionally assigned to the relevant terms. The terms "single-organism metabolic process", "membrane", and "catalytic activity" were dominant in each of the three main categories, respectively (Fig. A2). By comparing $\mathrm{CB}$ and $\mathrm{CA}, 1550$ differentially expressed genes, including 723 downregulated genes and 827 up-regulated genes, the terms "cellular metabolic process", "membrane", and "cation binding" were dominant in each of the three main categories, respectively (Fig. A3). "membrane" and "single-organism metabolic process" were mainly terms in the low temperature treatment.

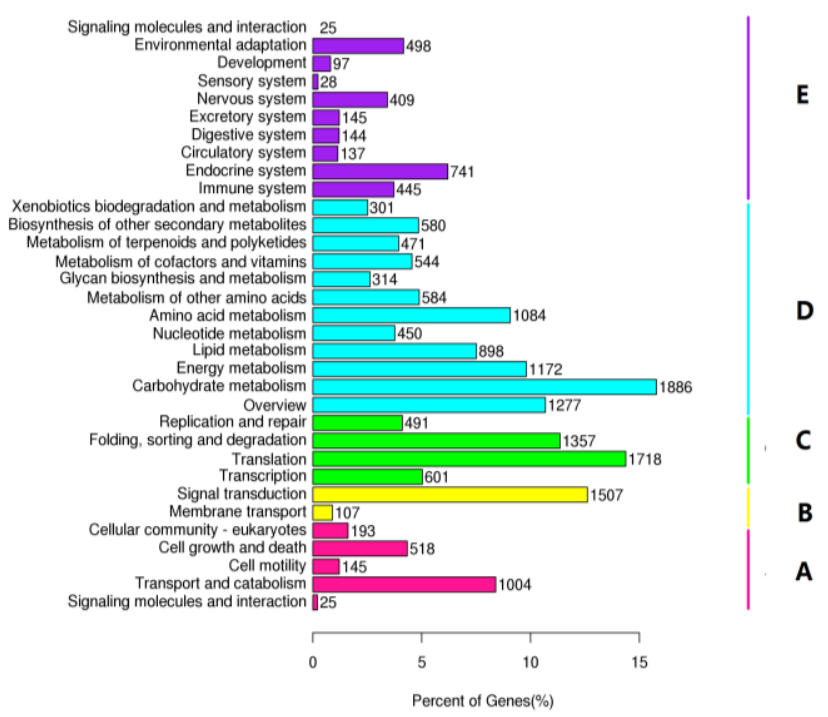

Figure 6. Kyoto Encyclopedia of Genes and Genomes (KEGG) functional classification of the unigenes $(\boldsymbol{A})$ cellular processes; $(\boldsymbol{B})$ environmental information processing; $(\boldsymbol{C})$ genetic information processing; $(\boldsymbol{D})$ metabolism; $(\boldsymbol{E})$ organismal systems 

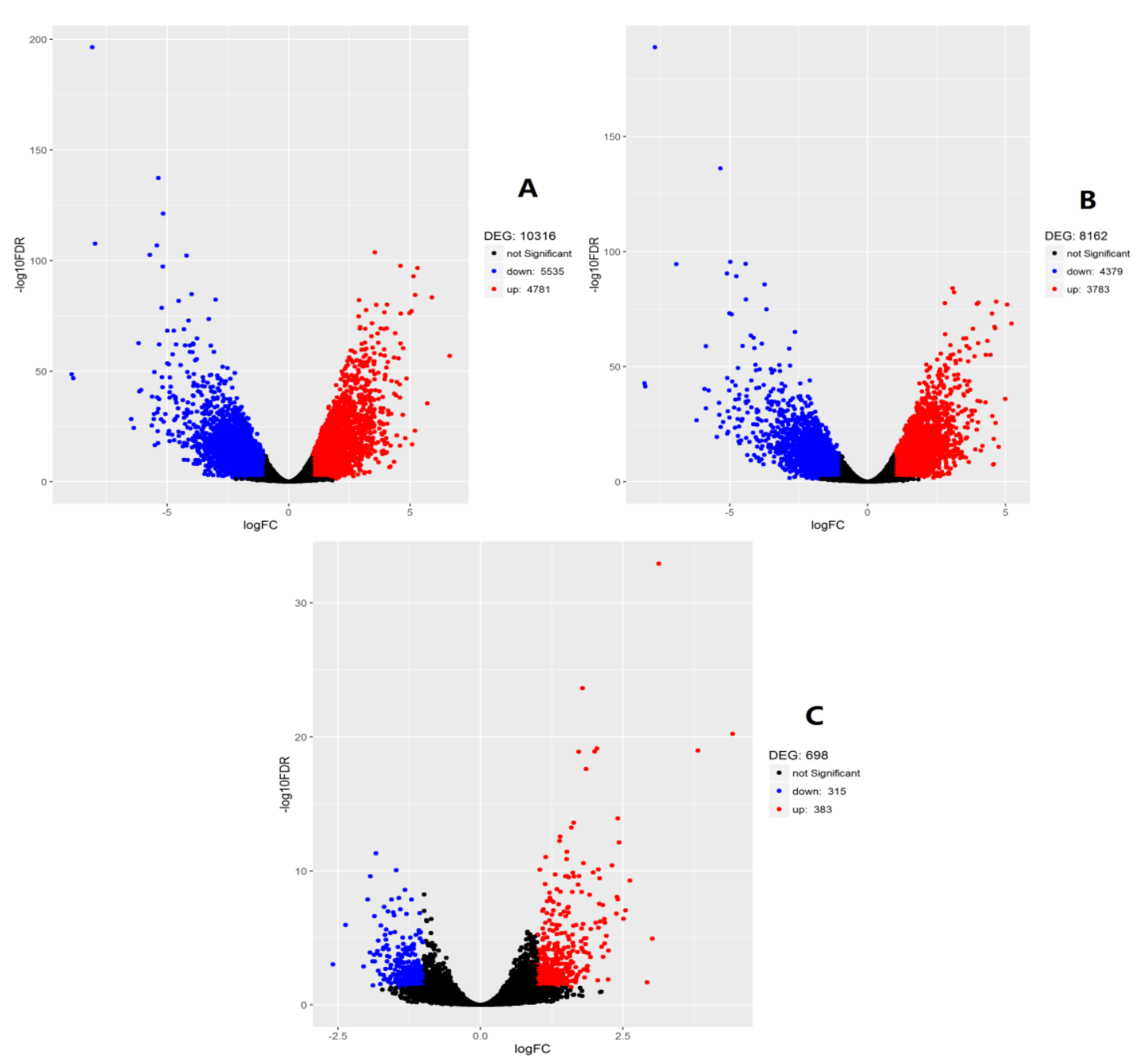

Figure 7. Volcano plot of differentially expressed genes at low temperature condition (A) $C A$ versus $C K$; $(\boldsymbol{B}) C B$ versus $C K$; $(\boldsymbol{C}) C B$ versus $C A$

\section{KEGG pathway analysis of the differentially expressed genes}

To determine whether the low temperature responsive genes engaged in specific pathways, the differentially expressed genes were used as objects to search against the KEGG pathway database. The top 10 pathways of the number of differentially expressed genes are counted. By comparing CA with CK, more differentially expressed genes were enriched in this 10 pathway, including 215 differentially expressed genes were related to "Carbon metabolism", 205 differentially expressed genes were related to "Ribosome", and 170 differentially expressed genes were related to "Biosynthesis of amino acids". "Plant hormone signal transduction (131)" and "Amino sugar and nucleotide sugar metabolism (123)" are obviously enriched, and the p-value is 1.51E-07 and 0.000946, respectively (Table 4). By comparing CB with CK, similar to the results of comparing CA with CK, 201 differentially expressed genes were related to "Carbon metabolism", 166 differentially expressed genes were related to "Ribosome", and 156 differentially expressed genes were related to "Biosynthesis of amino acids". "Plant hormone signal transduction (124)" and "Amino sugar and nucleotide sugar metabolism (115)" are obviously enriched, and the p-value is 4.40E-08 and 0.000561, respectively 
(Table 5). By comparing CB with CA, 20 differentially expressed genes were related to "Carbon metabolism", 18 differentially expressed genes were related to "Circadian rhythm-plant", and 14 differentially expressed genes were related to "Carbon fixation in photosynthetic organisms". "Circadian rhythm-plant (18)" and "Inositol phosphate metabolism (10)" are obviously enriched, and the p-value is 3.56E-12 and 0.001285, respectively (Table 6).

Table 4. KEGG enrichment analysis of differentially expressed genes between CA and CK

\begin{tabular}{|c|c|c|c|c|}
\hline Pathway ID & Pathway & KEGG pathway & $\begin{array}{l}\text { Enriched } \\
\text { genes }\end{array}$ & p-value \\
\hline ko01200 & Metabolism & Carbon metabolism & 215 & 0.570662 \\
\hline ko03010 & $\begin{array}{l}\text { Genetic information } \\
\text { Processing }\end{array}$ & Ribosome & 205 & 0.918432 \\
\hline ko01230 & Metabolism & Biosynthesis of amino acids & 170 & 0.965586 \\
\hline ko04141 & $\begin{array}{l}\text { Genetic information } \\
\text { processing }\end{array}$ & $\begin{array}{l}\text { Protein processing in } \\
\text { endoplasmic reticulum }\end{array}$ & 149 & 0.383433 \\
\hline ko04075 & $\begin{array}{l}\text { Environmental information } \\
\text { processing }\end{array}$ & $\begin{array}{l}\text { Plant hormone signal } \\
\text { transduction }\end{array}$ & 131 & $1.51 \mathrm{E}-07$ \\
\hline ko00500 & Metabolism & $\begin{array}{l}\text { Starch and sucrose } \\
\text { metabolism }\end{array}$ & 124 & 0.831474 \\
\hline ko00520 & Metabolism & $\begin{array}{l}\text { Amino sugar and nucleotide } \\
\text { sugar metabolism }\end{array}$ & 123 & 0.000946 \\
\hline ko03040 & $\begin{array}{l}\text { Genetic information } \\
\text { processing }\end{array}$ & Spliceosome & 119 & 0.304011 \\
\hline ko04144 & Cellular Processes & Endocytosis & 108 & 0.038656 \\
\hline ko00940 & Metabolism & $\begin{array}{l}\text { Phenylpropanoid } \\
\text { biosynthesis }\end{array}$ & 108 & 0.254553 \\
\hline
\end{tabular}

Table 5. KEGG enrichment analysis of differentially expressed genes between $C B$ and $C K$

\begin{tabular}{|c|c|c|c|c|}
\hline Pathway ID & Pathway & KEGG pathway & $\begin{array}{l}\text { Enriched } \\
\text { genes }\end{array}$ & p-value \\
\hline ko01200 & Metabolism & Carbon metabolism & 201 & 0.420369 \\
\hline ko03010 & $\begin{array}{l}\text { Genetic information } \\
\text { processing }\end{array}$ & Ribosome & 166 & 0.997957 \\
\hline ko01230 & Metabolism & Biosynthesis of amino acids & 156 & 0.950114 \\
\hline ko04141 & $\begin{array}{l}\text { Genetic information } \\
\text { processing }\end{array}$ & $\begin{array}{l}\text { Protein processing in } \\
\text { endoplasmic reticulum }\end{array}$ & 139 & 0.285677 \\
\hline ko04075 & $\begin{array}{l}\text { Environmental information } \\
\text { processing }\end{array}$ & $\begin{array}{l}\text { Plant hormone signal } \\
\text { transduction }\end{array}$ & 124 & $4.40 \mathrm{E}-08$ \\
\hline ko00520 & Metabolism & $\begin{array}{l}\text { Amino sugar and nucleotide } \\
\text { sugar metabolism }\end{array}$ & 115 & 0.000561 \\
\hline ko00500 & Metabolism & Starch and sucrose metabolism & 112 & 0.842905 \\
\hline ko00940 & Metabolism & Phenylpropanoid biosynthesis & 110 & 0.035962 \\
\hline ko03040 & $\begin{array}{l}\text { Genetic information } \\
\text { processing }\end{array}$ & Spliceosome & 100 & 0.633846 \\
\hline ko00230 & Metabolism & Purine metabolism & 95 & 0.229666 \\
\hline
\end{tabular}


Table 6. KEGG enrichment analysis of differentially expressed genes between $C B$ and $C A$

\begin{tabular}{|c|c|c|c|c|}
\hline Pathway ID & Pathway & KEGG pathway & $\begin{array}{c}\begin{array}{c}\text { Enriched } \\
\text { genes }\end{array} \\
\end{array}$ & p-value \\
\hline ko01200 & Metabolism & Carbon metabolism & 20 & 0.071514 \\
\hline ko04712 & Organismal systems & Circadian rhythm - plant & 18 & $3.56 \mathrm{E}-12$ \\
\hline ko00710 & Metabolism & $\begin{array}{c}\text { Carbon fixation in } \\
\text { photosynthetic organisms }\end{array}$ & 14 & 0.002501 \\
\hline ko04075 & $\begin{array}{l}\text { Environmental information } \\
\text { processing }\end{array}$ & $\begin{array}{l}\text { Plant hormone signal } \\
\text { transduction }\end{array}$ & 13 & 0.002586 \\
\hline ko04141 & Genetic information processing & $\begin{array}{l}\text { Protein processing in } \\
\text { endoplasmic reticulum }\end{array}$ & 13 & 0.146036 \\
\hline ko00010 & Metabolism & Glycolysis/Gluconeogenesis & 11 & 0.105862 \\
\hline ko00562 & Metabolism & Inositol phosphate metabolism & 10 & 0.001285 \\
\hline ko00564 & Metabolism & $\begin{array}{l}\text { Glycerophospholipid } \\
\text { metabolism }\end{array}$ & 10 & 0.009997 \\
\hline ko04626 & Organismal systems & Plant-pathogen interaction & 9 & 0.059561 \\
\hline ko04120 & Genetic information processing & Ubiquitin mediated proteolysis & 9 & 0.082081 \\
\hline
\end{tabular}

\section{Transcription factors}

Transcription factors (TFs) are important upstream regulatory proteins and play significant roles in plant development and tolerance (Chinnusamy et al., 2010). In this study, a total of 1130 unigenes were identified to be involved in transcription. By comparing CA and CK, 499 differentially expressed genes (219 up-regulated and 280 down-regulated) were identified. The largest gene family was the bHLH family, followed by ERF, C3H, MYB-related, C2H2, and bZIP family (Fig. 8A). By comparing CB and CK, 453 differentially expressed genes (197 up-regulated and 256 downregulated) were identified. The largest gene family was the bHLH family, followed by ERF, C3H, MYB-related, C2H2, and bZIP family (Fig. 8B). By comparing CB and CA, 55 differentially expressed genes (28 up-regulated and 27 down-regulated) were identified. The largest gene family was the WRKY family, followed by MYB-related, bHLH, bZIP, and ERF family (Fig. 8C).
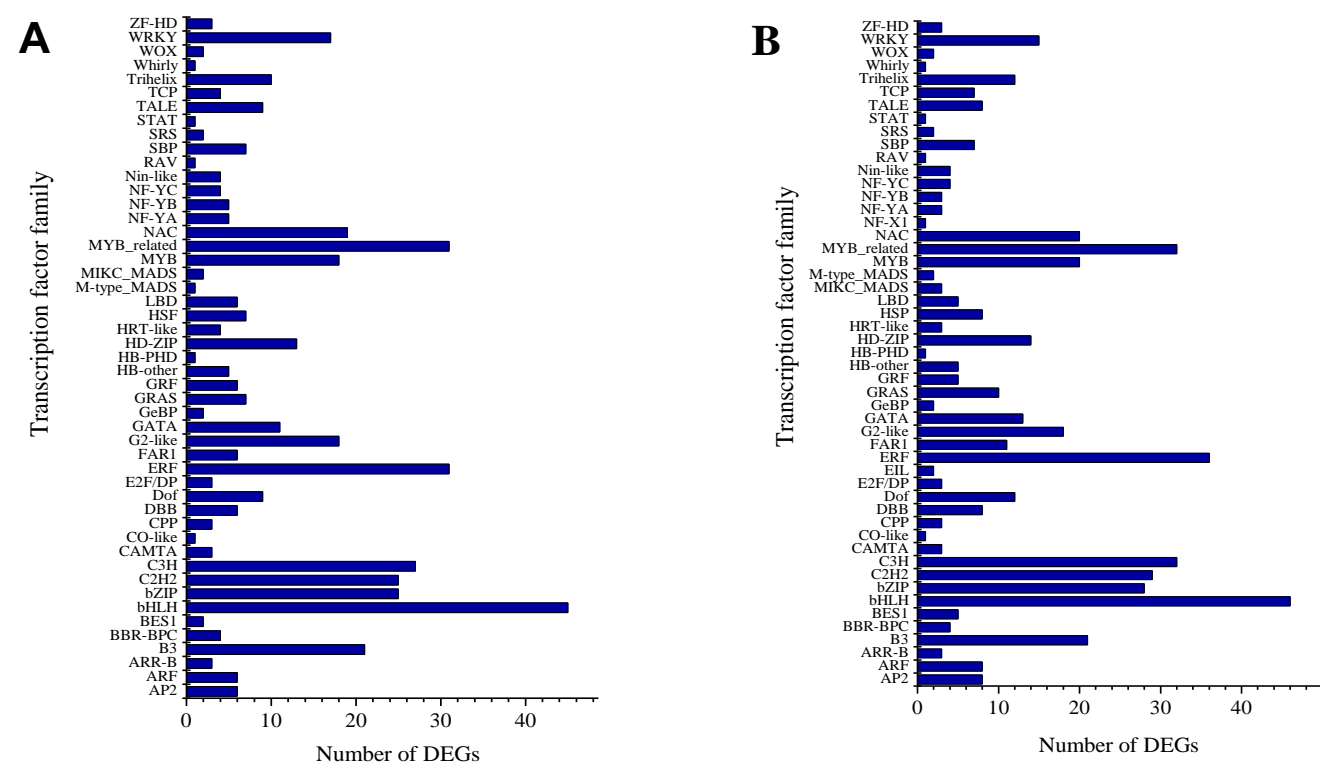


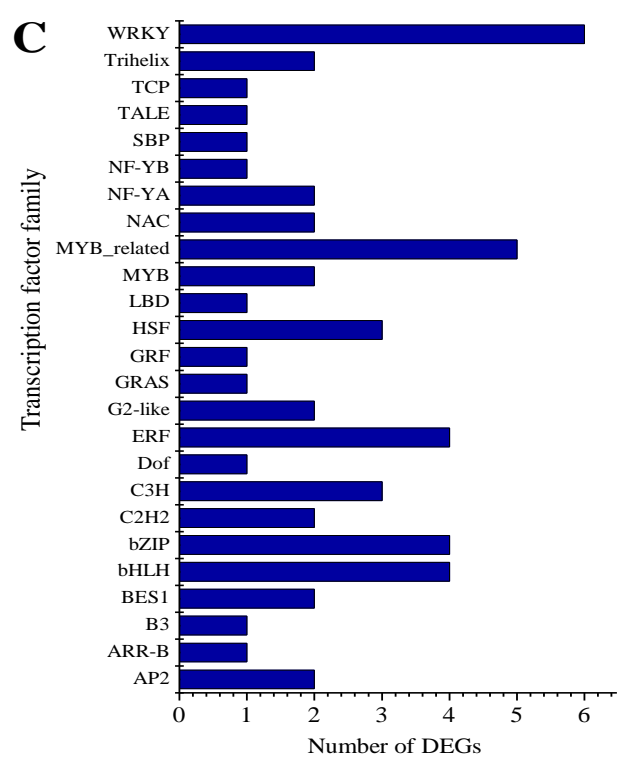

Figure 8. Distribution of differentially expressed genes in transcription factor families $(\boldsymbol{A}) C A$ versus $C K ;(\boldsymbol{B}) C B$ versus $C K$; $(\boldsymbol{C}) C B$ versus $C A$

\section{Genes expression analysis by $q R T-P C R$}

To confirm the reliability of the RNA-Seq data, real-time quantitative reverse transcription PCR was used to assess the expression level of 6 selected genes which were significantly affected at low temperature. These 6 selected differentially expressed genes are all transcription factors, including bZIP, NAC, HSF, C2H2, LBD and NF-YA, most of them are known to be related to low temperature. Results compared with the sequencing results, although the variation of gene was different, all the unigenes showed consistent expression patterns that were consistent with the RNA-Seq data, indicating that our experimental results were valid (Fig. 9).

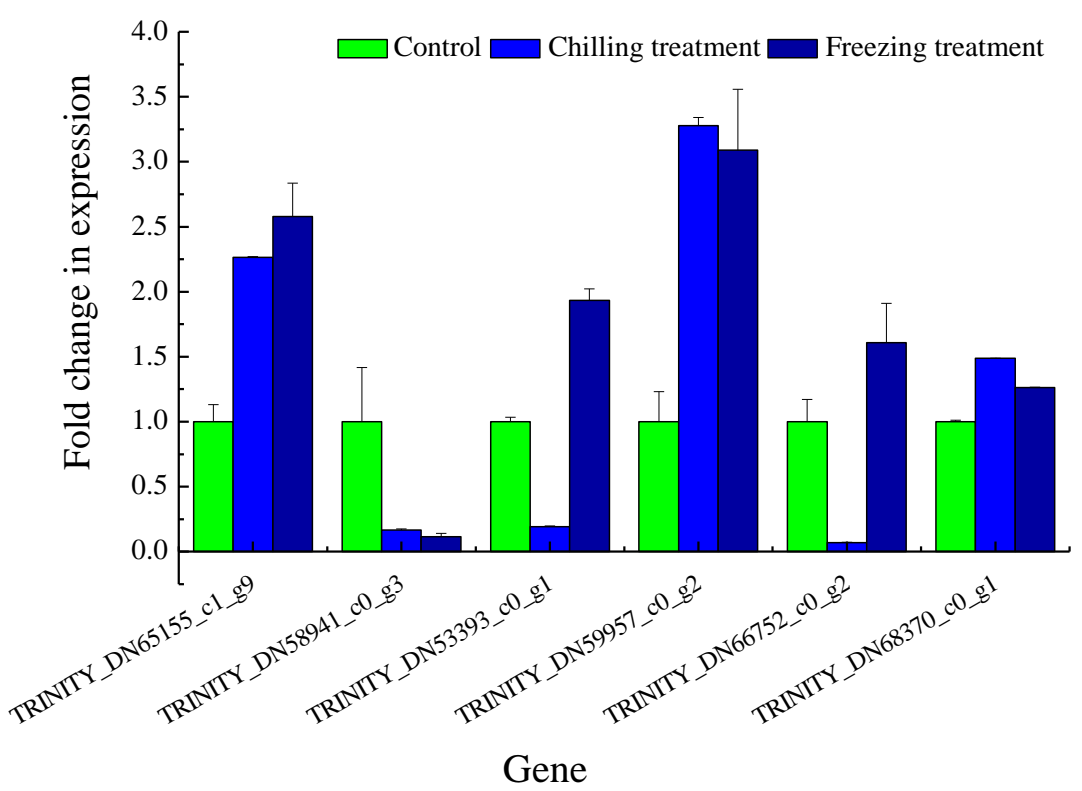

Figure 9. Quantitative real-time PCR analysis of six differently expressed genes 


\section{Discussion}

H. ammodendron, as an important component of old Mediterranean flora, is widely spread in the Junggar basin, northeast of the Tarim basin and other desert areas on a range of soil and is a representative psammophytic species (Wang et al., 2014). It is a precious biological resource in the arid desert region. In Junggar basin, most of the desert species can utilize meltwater to germinate in early spring, it is available for better germination and for seedling survival before the desiccation period (Huang et al., 2009). Wang research showed that $H$. ammodendron can germinate when the temperature close to $0^{\circ} \mathrm{C}$ (Wang et al., 2006), and Zhang showed that the germination percentage of $H$. ammodendron and Haloxylon Persicum were over $80 \%$ under $2{ }^{\circ} \mathrm{C}$ (Zhang, 2010). In addition, Sun suggested desert seed start to germinate only two days when snow melted at the average daily temperature (day/night) of $3.5^{\circ} \mathrm{C}$ (Sun, 2015). Therefore, it is of great significance to study the molecular mechanism of $H$. ammodendron germination at low temperature condition. The cold resistance of plants is a quantitative trait controlled by multiple genes, and a large number of genes are involved in the mechanism of low temperature. H. ammodendron has formed a complex mechanism to resist low temperature. When receiving cold signals, some genes are differentially expressed to enhance cold resistance, and some plants will produce a series of reactions, such as morphological level, physiological and biochemical level and molecular level. A transcriptional analysis of Arabidopsis thaliana (Norma et al., 2008), wheat (Gulick et al., 2005) and sunflower (Airaki et al., 2012) at low temperature showed that a large number of genes related to photosynthesis were significantly inhibited at low temperature.

By comparing the effects of low temperature on seed germination, we identified 17995, 15511 and 1550 differentially expressed genes in the comparisons of chilling treatment versus control, freezing treatment versus control and freezing treatment versus chilling treatment, respectively. Notably, more genes were differentially expressed under chilling treatment versus control, freezing treatment versus control, indicating that the alteration in gene expression at the cold condition, they will generate a large number of genes to adapt. The number of differentially expressed genes is relatively less under freezing treatment versus chilling treatment, suggesting that chilling treatment and freezing treatment are similar in response to low temperature, and plants are sensitive to low temperature. Moreover, a total of 607 genes overlapped with those of chilling treatment versus control, freezing treatment versus control and freezing treatment versus chilling treatment, indicating a linkage among three comparisons and a progressive biological process. These differentially expressed genes were mapped to each term of the GO database, "single-organism metabolic process" and "membrane" was strongly affected at low temperature condition, and the plasma membrane is believed to be the primary site in response to low temperature signal. Through the analysis of the KEGG pathway, the top 10 pathways were basically same in chilling treatment versus control and freezing treatment versus control, including "Metabolism", "Environmental Information Processing" and "Genetic Information Processing" pathway. "Plant hormone signal transduction" was significantly enriched in chilling treatment versus control, freezing treatment versus control and freezing treatment versus chilling treatment. A lot of research has proven that plant hormones play an important role in resistance of plants (Nakashima et al., 2014). In this study, 131, 124, 13 differentially expressed genes were found to participate in plant hormone signal transduction. 
Membrane systems, which are known to be the primary site of freezing injury in plants, suffer multiple forms of damage caused by freeze-induced cellular dehydration (Kawamura et al., 2003). Membranes must be kept fluid in order to sustain the functional activity of membrane proteins and membranes themselves at low temperature condition (Zhou et al., 2010). An important performance of many plants is to increase unsaturated fatty acids, such as increasing the proportion of oleic acid, linoleic acid and linolenic acid in total fatty acids (Chen et al., 2005). In this study, we identified 3 lipid transfer proteins (LTP) genes and 4 fatty acid desaturase (FAD) genes. Among these, 2 LTP genes and 2 FAD genes were up-regulated, 1 LTP gene and 2 FAD genes were down-regulated at low temperature condition. A series of changes in gene expression will occur in plants, and a large number of genes are induced at low temperature. Some proteins encoded by these genes are so-called anti-freezing proteins (AFPs), including late-embryogenesis-abundant proteins (LEA), the LEA protein functions as an antioxidant, as well as a membrane and protein stabilizer (Tunnacliffe and Wise, 2007). AFPs also include thaumatin-like proteins (TLPs), polygalacturonase-inhibitor proteins (PGIPs), heat-shock proteins (HSPs), etc. Proline is one of the most important organic osmolytes, and participates in the responses to various environmental (Ashraf and Foolad, 2007). The increase of proline is considered as one of the mechanisms of cold resistance, delta-1-pyrroline-5-carboxylate synthase (P5CS) plays a key role in proline biosynthesis, it is involved in osmoregulation and abiotic tolerance in plants (Xia et al., 2014). Szekely shows that P5CS participates in the cold-responsive and shows high expression (Szekely et al., 2008). In our study, we found 1 TLP, 5 PGIPs, 2 LEAs were up-regulated at low temperature, and these genes play an important role to tolerate low temperatures. Contrary to previous research, all genes encoding P5CS were to be significantly down-regulated at low temperature, and this may be the difference displayed by different species.

As an important second messenger, $\mathrm{Ca}^{2+}$ was reported play a role in regulating growth processes and cold signaling (Reddy et al., 2011), and it was sensed by proteins of three main classes at low temperature: $\mathrm{Ca}^{2+}$ dependent protein kinases (CDPKs), cell adhesion molecule (CaMs) and calcineurin B-like protein (CBL) (Miura and Furumoto, 2013; Boudsocq and Sheen, 2013). A further important pathway is the mitogenactivated protein kinases (MAPKs) cascade (Chinnusamy et al., 2010). Wang et al. showed that CDPKs, calmodulin, CBL, MAPKs were involved in signal transduction upon low temperature in Camellia sinensis (Wang et al., 2013). In this study, we identified 4 CDPK genes, 6 MAPK genes, 6 CBL genes and 3 calmodulin genes were up-regulated at low temperature. Yang et al. found that the calcium/calmodulinregulated receptor kinase (CRLK1) was crucial for cold tolerance in plants (Yang et al., 2010). 1 CRLK1 gene was identified to be up-regulated in this study, meanwhile, we found that 3 CRLK2 were obviously up-regulated at low temperature. Therefore, CRLK2 may be important to tolerate low temperature.

Transcriptional regulation is a key step for plants to adapt the changes of environment, and transcription factors play an important role in the plant. In this study, it was found that many transcription factors have changed at low temperature. Among these TFs, most of the family has been reported to be linked to cold resistance in plants (Rushton et al., 2010; Zhang et al., 2012; Hu et al., 2008; Fode et al., 2008). A large number of transcription factors showed abundant diversity, and some genes in same transcription factor family had both up-regulated and down-regulated genes. The change of expression level of specific transcription factors can greatly affect the ability of 
plants to adapt to adversity. The results of this study indicate that low temperature can induce or inhibit the expression of many transcription factors. Among them, bHLH, ERF, C3H, MYB-related, C2H2, and bZIP were the most numerous and significant transcription factor families in chilling treatment versus control and freezing treatment versus control. By comparing freezing treatment and chilling treatment, the lower temperature increased the number of other transcription factor families, which could regulate lower temperature condition. Pang et al. showed that ERF and WRKY transcription factors were up-regulated in seedlings treated at $4{ }^{\circ} \mathrm{C}$ for 14 days (Pang et al., 2013). High salt and low temperature could induce the expression of 3 WRKYs genes in grape, indicating that WRKYs was involved in the process of grape resistance to low temperature (Hou et al., 2013). In this study, the ERF (Fold changes from 0.44 to 4.06) and WRKYs (Fold changes from 0.75 to 1.17) of $H$. ammodendron were identified to be up-regulated at low temperatures, these results indicated that they are important regulators of $H$. ammodendron in response to low temperature. Six transcription factors were selected for further expression analysis, and they have been identified by qRT-PCR showing the same trend as our Illumina sequencing.

\section{Conclusions}

Haloxylon ammodendron, as one of the dominant species in the desert area of China, has strong low temperature tolerance. In this study, we present a considerable portion of the transcriptome of $H$. ammodendron after low temperature using RNA-seq and differentially expressed gene analyses. Based on the assembled de novo transcriptome, a large number of differentially expressed genes were identified, and include many candidate genes involved in low temperature By performing BLAST analysis of the all unigenes against public databases ( $\mathrm{Nr}, \mathrm{KOG}, \mathrm{GO}$ and $\mathrm{KEGG}$ ), we obtained functional annotations and classifications. Moreover, a large number of transcription factors were identified under low temperature. This represents a fully characterized transcriptome, and provides a valuable resource for genetic and genomic studies in Haloxylon ammodendron. It could also help to explore the cold-related genes in improving the understanding of low-temperature tolerance and plant-environment interactions.

Acknowledgements. The research was supported by the Program of the National Natural Science Foundation of China (31570595, 31660194), General Financial Grant of the China Postdoctoral Science Foundation (2017M613253) and Scientific Research Foundation of Shihezi University for Advanced Talents (RCZX201518, RCZX201521).

\section{REFERENCES}

[1] Airaki, M., Leterrier, M., Mateos, R. M., Valderrama, R., Chaki, M., Barroso, J. B. (2012): Metabolism of reactive oxygen species and reactive nitrogen species in pepper (Capsicum annuum l.) plants under low temperature stress. - Plant Cell and Environment 35(2): 281-295.

[2] Ashraf, M., Foolad, M. R. (2007): Roles of glycine betaine and proline in improving plant abiotic stress resistance. - Environmental and Experimental Botany 59(2): 206-216.

[3] Avagyan, V. (2009): BLAST+: architecture and applications. - BMC Bioinformatics 10(1): $1-9$. 
[4] Bateman, A., Birney, E., Cerruti, L., Durbin, R., Etwiller, L., Eddy, S. R. (2000): The pfam protein families database. - Nucleic Acids Research 28(1): 263-266(4).

[5] Boudsocq, M., Sheen, J. (2013): CDPKs in immune and stress signaling. - Trends in Plant Science 18(1): 30-40.

[6] Cai, H., Tian, S., Liu, C., Dong, H. (2011): Identification of a MYB3R gene involved in drought, salt and cold stress in wheat (Triticum aestivum l.). - Gene 485(2): 146-152.

[7] Chen, J., Tian, Q., Pang, T., Jiang, L., Wu, R., Xia, X. (2014): Deep-sequencing transcriptome analysis of low temperature perception in a desert tree, Populus Euphratica. - Bmc Genomics 15(1): 326.

[8] Chen, N., Guo, S. J., Meng, Q. W. (2005): Relationship between plant chilling tolerance and membrane lipidscomposition and its advances in researches on molecular biology. Biotechnology Bulletin 21(2): 6-29.

[9] Cheng, Z. Y., Li, M., Shi, Y., He, P., He, L. L., Li, F. S. (2015): Research of droughtresponse mechanism in sugarcane by high throughput sequencing-based digital gene expression profiling. - Molecular Plant Breeding 13(9): 2018-2028.

[10] Chinnusamy, V., Zhu, J. K., Sunkar, R. (2010): Gene regulation during cold stress acclimation in plants. - Plant Stress Tolerance 639: 39-55.

[11] Conesa, A., Götz, S., García-Gómez, J. M. (2005): Blast2GO: a universal tool for annotation, visualization and analysis in functional genomics research. - Bioinformatics 21(18): 3674-6.

[12] Consortium, U. P. (2015): UniProt: a hub for protein information. - Nucleic Acids Research 43(Database issue): D204-12.

[13] Fode, B., Siemsen, T., Thurow, C., Weigel, R., Gatz, C. (2008): The Arabidopsis GRAS protein SCL14 interacts with class II TGA transcription factors and is essential for the activation of stress-inducible promoters. - Plant Cell 20: 3122-3135.

[14] Grabherr, M. G., Haas, B. J., Yassour, M., Levin, J. Z., Thompson, D. A., Amit, I. (2011): Full-length transcriptome assembly from rna-seq data without a reference genome. - Nature Biotechnology 29(7): 644.

[15] Gulick, P. J., Drouin, S., Yu, Z., Danyluk, J., Poisson, G., Monroy, A. F. (2005): Transcriptome comparison of winter and spring wheat responding to low temperature. Genome 48(48): 913-923.

[16] Haas, B. J., Papanicolaou, A., Yassour, M. (2013): De novo transcript sequence reconstruction from RNA-seq using the Trinity platform for reference generation and analysis. - Nature Protocols 8(8): 1494-1512.

[17] Han, J. X., Wei, Y., Yan, C., An, S. Z. (2011): The vivipary characteristic of Anabasis elatior and its ecological adaptation. - Acta Ecologica Sinica 31(10): 2662-2668.

[18] Harris, M. A., Clark, J., Ireland, A. (2004): The Gene Ontology (GO) database and informatics resource. - Nucleic Acids Research 32(suppl_1): D258-61.

[19] Hou, L., Wang, W. J., Guo, X. P., Fu, P. N., Liu, X. (2013): Gene cloning and expression analysis of three WRKYs in Vitis vinifera L. - Plant Physiology Journal 49(3): 289-296.

[20] Hu, H., You, J., Fang, Y., Zhu, X., Qi, Z., Xiong, L. (2008): Characterization of transcription factor gene SNAC2 conferring cold and salt tolerance in rice. - Plant Molecular Biology 67(1-2): 169-181.

[21] Huang, P. Y. (2002): Non irrigated vegetation and its restoration in Arid Areas. - Beijing: Science Press 123-134.

[22] Huang, P. Y., Xiang, B., Qi-Jian, L. I., Ze-Hai, X. U. (2009): Relationship between Haloxylon ammodendron seedling dynamics and habitat before summer. - Journal of Desert Research 29(1): 87-94.

[23] Huang, Z., Zhang, X., Zheng, G., Gutterman, Y. (2003): Influence of light, temperature, salinity and storage on seed germination of Haloxylon ammodendron. - Journal of Arid Environments 55(3): 453-464. 
[24] Kawamura, Y., Uemura, M. (2003): Mass spectrometric approach for identifying putative plasma membrane proteins of Arabidopsis leaves associated with cold acclimation. Plant Journal 36(2): 141-154.

[25] Li, H., Yao, W., Fu, Y., Li, S., Guo, Q. (2015): De novo assembly and discovery of genes that are involved in drought tolerance in tibetan Sophora moorcroftiana. - Plos One 10(1): e111054.

[26] Li, L., Zhang, X. M. (2007): Germination strategies of two halophytes in salt desert of northwestern china. - Science in China 50(1): 115-121.

[27] Livaka, K. J., Schmittgen, T. D. (2001): Analysis of relative gene expression data using real-time quantitative PCR and the $2^{-\Delta \Delta \mathrm{CT}}$ method. - Methods 25(4): 402-408.

[28] Miura, K., Furumoto, T. (2013): Cold signaling and cold response in plants. International Journal of Molecular Sciences 14(3): 5312-5337.

[29] Nakashima, K., Yamaguchi-Shinozaki, K., Shinozaki, K. (2014): The transcriptional regulatory network in the drought response and its crosstalk in abiotic stress responses including drought, cold, and heat. - Frontiers in Plant Science 5(170): 170.

[30] Norma, P., Esteban, H. H., Luis, F., Di, R. J., Paula, F., Heinz, R. A. (2008): Transcriptomic identification of candidate genes involved in sunflower responses to chilling and salt stresses based on cDNA microarray analysis. - BMC Plant Biology 8(1): 11 .

[31] Pang, T., Ye, C. Y., Xia, X., Yin, W. (2013): De novo sequencing and transcriptome analysis of the desert shrub, Ammopiptanthus mongolicus, during cold acclimation using Illumina/Solexa. - BMC Genomics 14(1): 488-488.

[32] Peng, M. W., Wang, M., Jiang, P., Chang, Y. L., Chu, G. M. (2018): The impact of low temperature on seed germination of two desert species in Junggar basin of China. Applied Ecology And Environmental Research 16(5): 5771-5780.

[33] Reddy, A. S., Ali, G. S., Celesnik, H., Day, I. S. (2011): Coping with stresses: roles of calcium-and calcium/calmodulin-regulated gene expression. - Plant Cell 23(6): 20102032.

[34] Rushton, P. J., Somssich, I. E., Ringler, P., Shen, Q. J. (2010): WRKY transcription factors. - Trends in Plant Science 15(5): 247-258.

[35] Sun, Y. Y. (2015): Drought Adaptation Characteristics of Plant Seedling Establishment in Junggar Desert. - Shihezi University, Chinese Shihezi.

[36] Szekely, G., Abraham, E., Cseplo, A., Rigo, G., Zsigmond, L., Csiszar, J., Ayaydin, F. (2008): Duplicated P5CS genes of Arabidopsis play distinct roles in stress regulation and developmental control of proline biosynthesis. - Plant Journal 53(1): 11-28.

[37] Tatusov, R. L., Fedorova, N. D., Jackson, J. D. (2003): The COG database: an updated version includes eukaryotes. - BMC Bioinformatics 4(1): 41.

[38] Tunnacliffe, A., Wise, M. J. (2007): The continuing conundrum of the LEA proteins. Naturwissenschaften 94(10): 791-812.

[39] Wang, M., Zhang, S., Chu, G. (2014): Point pattern analysis of different life stages of Haloxylon ammodendron in desert-oasis ecotone of south junggar basin. - Polish Journal of Environmental Studies 23(6): 2271-2277.

[40] Wang, T. T. (2018): Proteomics and metabolomics analysis to cold stress in two desert chenopodiaceae plants seedlings. - Shihezi University, Chinese Shihezi.

[41] Wang, X. C., Zhao, Q. Y., Ma, C. L., Zhang, Z. H., Cao, H. L., Kong, Y. M. (2013): Global transcriptome profiles of Camellia sinensis during cold acclimation. - BMC Genomics 14(1): 415-415.

[42] Wang, X. Y., Wei, Y., Yan, C. (2006): Study on the effects of thermoperiods and fruiting wings on the germination of Haloxylon ammodendron seeds. - Arid Zone Research 2016(4).

[43] Xia, Z., Wei, T., Jia, L., Liu, Y. (2014): Co-expression of rice OsP5CS1 and OsP5CS2 genes in transgenic tobacco resulted in elevated proline biosynthesis and enhanced abiotic 
stress tolerance. - Chinese Journal of Applied and Environmental Biology 20(4): 717 722.

[44] Xie, C., Mao, X., Huang, J., Ding, Y., Wu, J., Dong, S. (2011): Kobas 2.0: a web server for annotation and identification of enriched pathways and diseases. - Nucleic Acids Research 39(Web Server Issue): 316-22.

[45] Yang, T., Ali, G. S., Yang, L., Du, L., Reddy, A., Poovaiah, B. W. (2010): Calcium/calmodulin-regulated receptor-like kinase CRLK1 interacts with MEKK1 in plants. - Plant Signal Behav 5(8): 991-994.

[46] Zhang, L., Zhao, G., Jia, J., Liu, X., Kong, X. (2012): Molecular characterization of 60 isolated wheat MYB genes and analysis of their expression during abiotic stress. Journal of Experimental Botany 63(1): 203-214.

[47] Zhang, S. J. (2010): Study on Natural Cure of Main Plant Species at Initialized Process in the South Marginal Zone of the Junggar Basin. - Arid Environmental Monitoring 24: 8993.

[48] Zhou, Q. (2016): Transcriptome of Seed and DGE Method Research of Seed Low Temperature Germination Period in Lepidium apetalum willd. - Xinjiang Normal University, Xinjiang.

[49] Zhou, Z., Wang, M. J., Zhao, S. T., Hu, J. J., Lu, M. Z. (2010): Changes in freezing tolerance in hybrid poplar caused by up- and down-regulation of PtFAD2 gene expression. - Transgenic Research 19(4): 647-654.

\section{APPENDIX}

Figure A1. GO functional classification analysis of differentially expressed genes in $C A$ vs $C K$

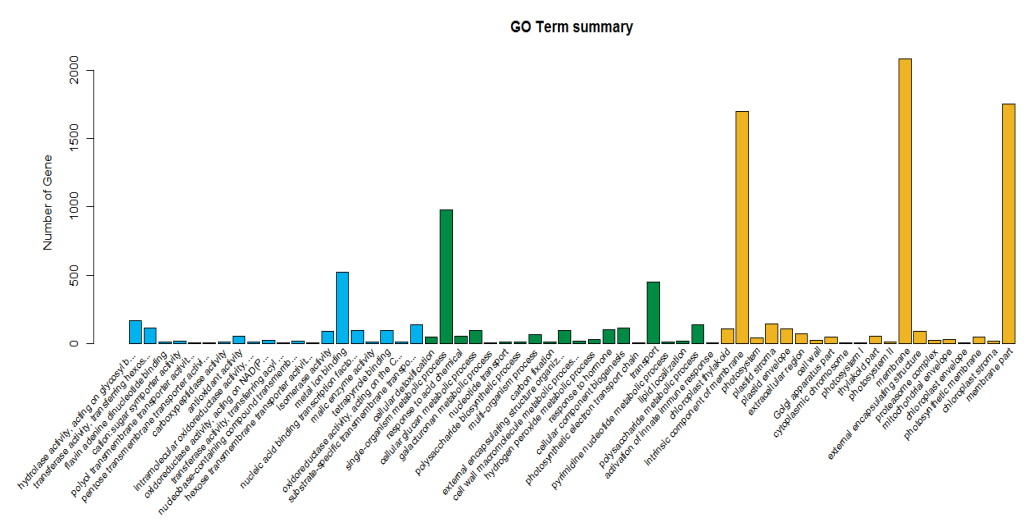

Figure A2. GO functional classification analysis of differentially expressed genes in $C B$ vs $C K$

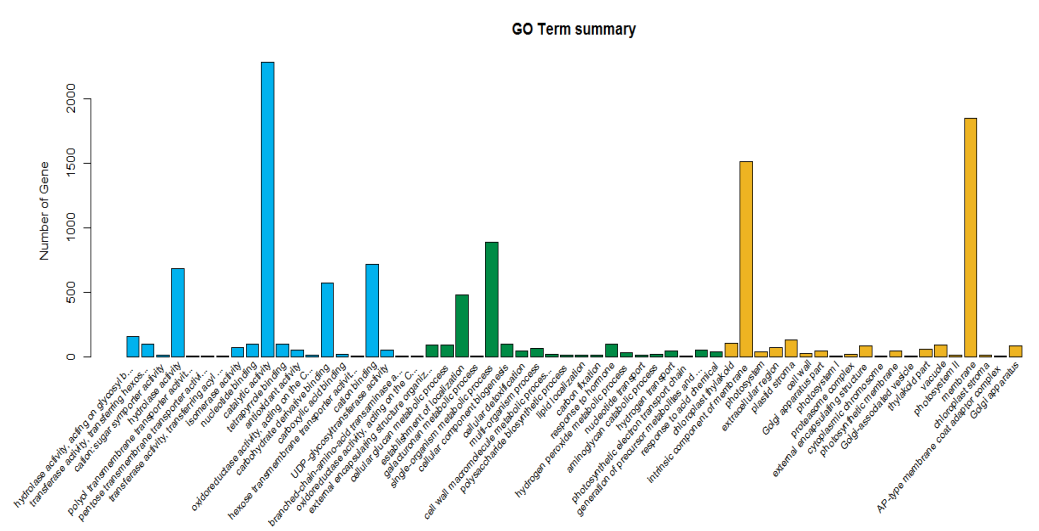


Figure A3. GO functional classification analysis of differentially expressed genes in $C B$ vs $C A$

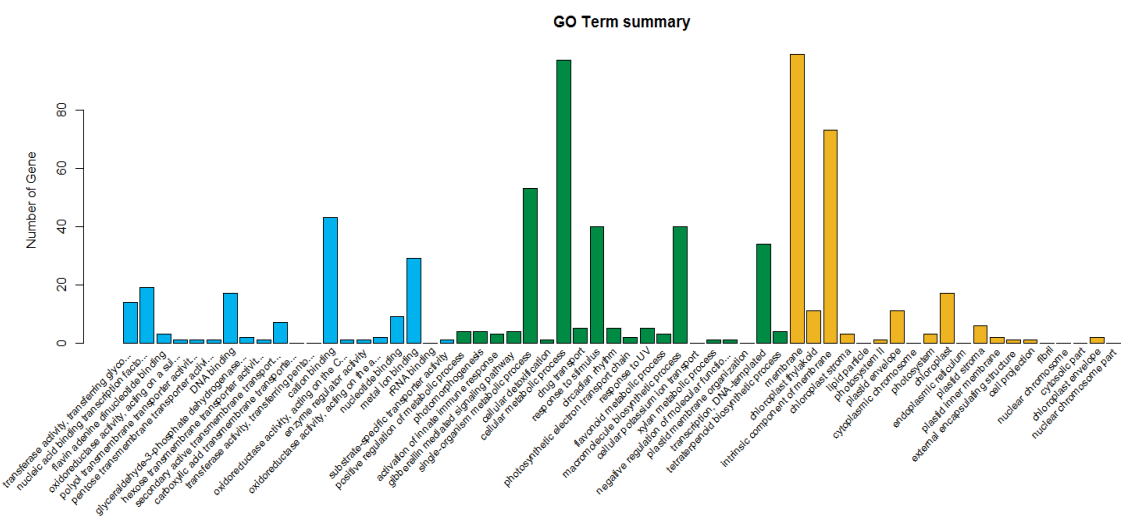

